\title{
Control of the TSU 2-m automatic telescope
}

\author{
Joel A. Eaton and Michael H. Williamson \\ Center of Excellence in Information Systems, Tennessee State University, \\ 330 Tenth Avenue North, Nashville, TN 30203 USA
}

\begin{abstract}
Tennessee State University is operating a 2-m automatic telescope for high-dispersion spectroscopy. The altazimuth telescope is fiber-coupled to a conventional echelle spectrograph with two resolutions $(\mathrm{R}=30,000$ and $70,000)$. We control this instrument with four computers running linux and communicating over ethernet through the UDP protocol. A computer physically located on the telescope handles the acquisition and tracking of stars. We avoid the need for real-time programming in this application by periodically latching the positions of the axes in a commercial motion controller and the time in a GPS receiver. A second (spectrograph) computer sets up the spectrograph and runs its CCD, a third (roof) computer controls the roll-off roof and front flap of the telescope enclosure, and the fourth (executive) computer makes decisions about which stars to observe and when to close the observatory for bad weather. The only human intervention in the telescope's operation involves changing the observing program, copying data back to TSU, and running quality-control checks on the data. It has been running reliably in this completely automatic, unattended mode for more than a year with all day-to-day adminsitration carried out over the Internet. To support automatic operation, we have written a number of useful tools to predict and analyze what the telescope does. These include a simulator that predicts roughly how the telescope will operate on a given night, a quality-control program to parse logfiles from the telescope and identify problems, and a rescheduling program that calculates new priorities to keep the frequency of observation for the various stars roughly as desired. We have also set up a database to keep track of the tens of thousands of spectra we expect to get each year.
\end{abstract}

Keywords: Telescope control, automation, quality control

\section{INTRODUCTION}

The Center of Excellence in Information Systems at Tennessee State University has been operating automatic telescopes for the past fifteen years, mostly moderate sized ones for aperture photometry. As part of our commitment to build and maintain a completely automatic observatory through which we can study astronomical phenomena simultaneously with imaging, spectroscopy, and photometry (http://schwab.tsuniv. edu), we have now added a 2-m telescope for high-dispersion spectroscopy (http://astro.tsuniv.edu) and may some day finish a moderate imaging telescope. All of these instruments operate autonomously under computer control at Fairborn Observatory (http://www.fairobs.org), a private site in southern Arizona.

Fig. 1 shows the 2-m Automatic Spectroscopic Telescope (AST) in its enclosure. We have designed it specifically for obtaining high-dispersion spectra of stars, since in our experience robotic telescopes work best when designed for only one particular type of observing. ${ }^{1}$ The telescope is a classical Cassegrain with an f/1.5 primary mirror and an aluminum secondary, turned by Oak Ridge National Laboratory, that gives an overall f/8 optical system. The focus is roughly 18 inches in front of the primary mirror. This placement reduces the required size of the secondary mirror to about 11 inches diameter with a weight of 10 pounds. It also means that the guiding head must be compact enough to fit into a relatively small space, which reduces the pressure to lard other instruments onto the telescope (see Fig. 2). The telescope acquires stars with a small video camera looking at the focus through a 45-degree pickoff mirror and some transfer lenses. A 200-micron fiber-optic cable projecting about 0.5 inches through the pickoff mirror takes starlight off to an echelle spectrograph in a separate enclosure south of the telescope. We guide on light spilling over the edge of the fiber optic cable. ${ }^{2}$ Fig. 3 shows an image

Further author information: J.A.E.: E-mail: eaton@donne.tsuniv.edu, Telephone: 16159637023

M.H.W.: E-mail: michael_h_williamson@yahoo.com, Telephone: 16159637012 


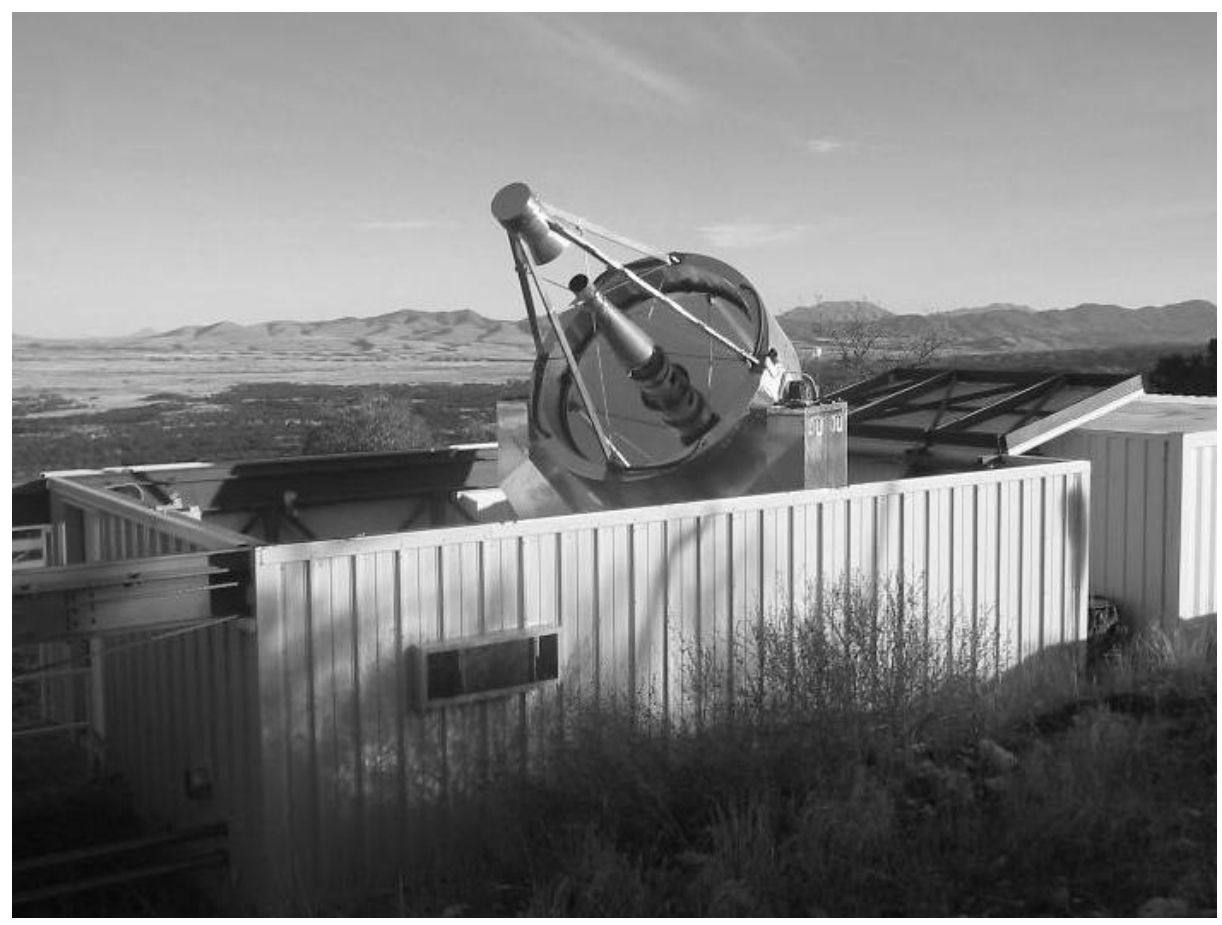

Figure 1. The TSU 2-m Automatic Spectroscopic Telescope. The telescope is housed in a barn-like enclosure with a top section that rolls off to the north. The echelle spectrograph is in an insulated shipping container to the south.

of a bright star on the fiber. We feed calibration light at $\mathrm{f} / 8$ into the spectrograph through a 600 -micron fiber, focusing its output onto the 200-micron fiber to the spectrograph (see Fig. 2).

The spectrograph itself (Fig. 4) is a rather conventional white-pupil design ${ }^{3,4}$ based on comercially available gratings. The camera is a custom design by Harland Epps, similar to the camera he disigned for the HobbyEberly Telescope ${ }^{5}$ but with some of the glasses changed to extend transmission into the near ultraviolet. This spectrograph has two wavelength settings chosen by rotating the cross-dispersion grating, a red position with wavelengths 5000-7100 $\AA$ and a blue one for 3700-5700 $\AA$. There is a standard resolution mode of approximately $\mathrm{R}=30,000$, based on feeding the spectrograph with a bare 200-micron fiber, and a $\mathrm{R}=70,000$ mode, based on feeding the spectrograph with a 75-micron slit in front of a second fiber. We record the spectra with a SITe ST-002A CCD, controlled with electronics package from Astronomical Research Cameras, Inc., at San Diego State University.

Because the telescope and its spectrograph must operate automatically, without anyone monitoring their performance in real time, the systems that control its various functions must be as reliable as absolutely possible. We are particularly concerned about interference between competing control processes - such as motion control and reading the CCD, about reliable communications between the various control processes, about graceful recoveries from various failures, and about protecting the equipment from physical damage. We also wanted to design a system based as much as possible on comercially available electronic components which could be replaced when they failed.

\section{RUNNING THE OBSERVATORY}

Fig. 5 shows the various computers that run the observatory. There are four of them connected through ethernet into a local network behind a firewall on a fifth, gateway, computer. In addition, we depend on periodic weather reports from another computer maintained by Fairborn Observatory. These various computers are distributed throughout the observatory to reduce the length of wiring to the devices they control. Communication among them is over conventional twisted-pair cables (RJ45) which seem to be quite reliable for this purpose and less 


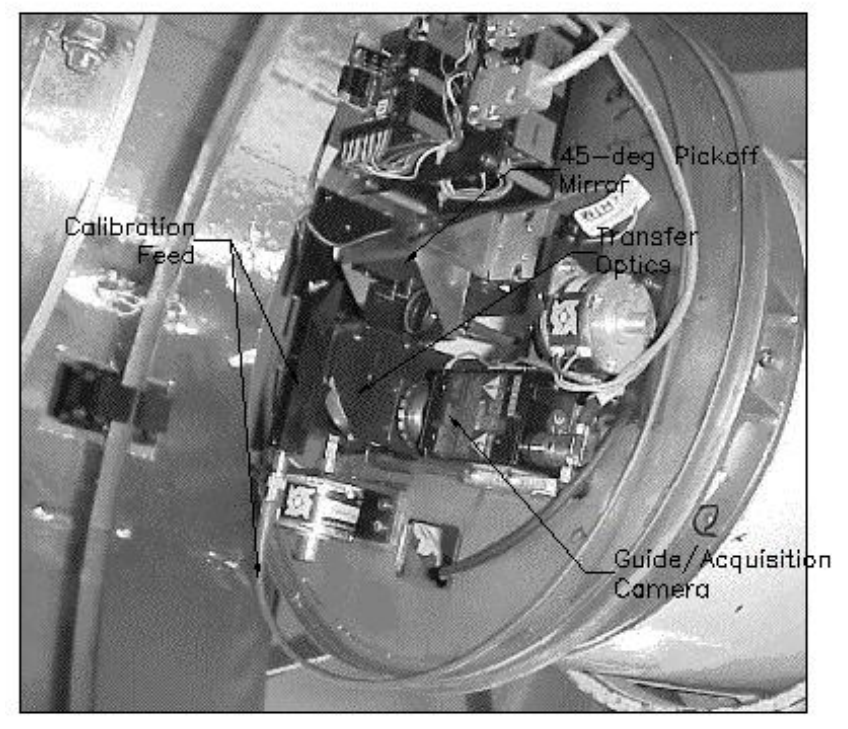

Figure 2. Guiding head in the TSU 2-m AST. If you look closely, you can see two fiber-optic cables projecting up through the pickoff mirror. The calibration feed consists of a fiber cable, to bring light up to the guiding head, and some reimaging optics (a lens and 45-deg prism in the long black cylinder, to focus light at f/8 onto an input fiber for the spectrograph. These fibers move under the calibration feed on a linear slide.

Figure 3. Image of a bright star centered on optical fiber.

susceptible to damage by lightning and power surges than coaxial cable. ${ }^{6}$ We also note that at least one factory in middle Tennessee uses this same setup for its production control system.

\subsection{Overall control and communications}

We run this observatory with four control programs, running as daemons on the four computers. Overall scheduling and coordination is through a process running on the executive computer, t13x. This daemon starts each night as a cron job, although it restarts automatically whenever the computer reboots. This executive process 1) monitors the status of the weather as provided by Fairborn Observatory, 2) tells the roof computer, t13r, when to open and close the telescope enclosure on the basis of time or weather conditions, 3 ) chooses stars to observe based on either a calculated priority or a specific time of observation, 4) orders the telescope computer, t13a, to acquire and track a star, 5) tells the spectrograph computer, t13c, to make a particular exposure, and 6) monitors these other computers for error/completion messages.

The control processes that manage the telescope, spectrograph, and main enclosure similarly operate as continuously running daemons on t13a, t13c, and t13r, respectively. They listen for commands from t13x and, in turn, send it completion/status messages, although t13a will stow the telescope on its own authority if it thinks it has lost contact with t13x. These various control processes obviously have to be coordinated to make any observations. We do this in two ways, 1) by writing status files through file-transfer protocol, ftp, to the disc of the appropriate computer and 2) by sending control messages directly between processes through ethernet. Writing files to disc is effective for transmitting information, such as weather status and roof status/control, that needs communicating only slowly, e.g., once a minute. For the more critical communications over ethernet, we 


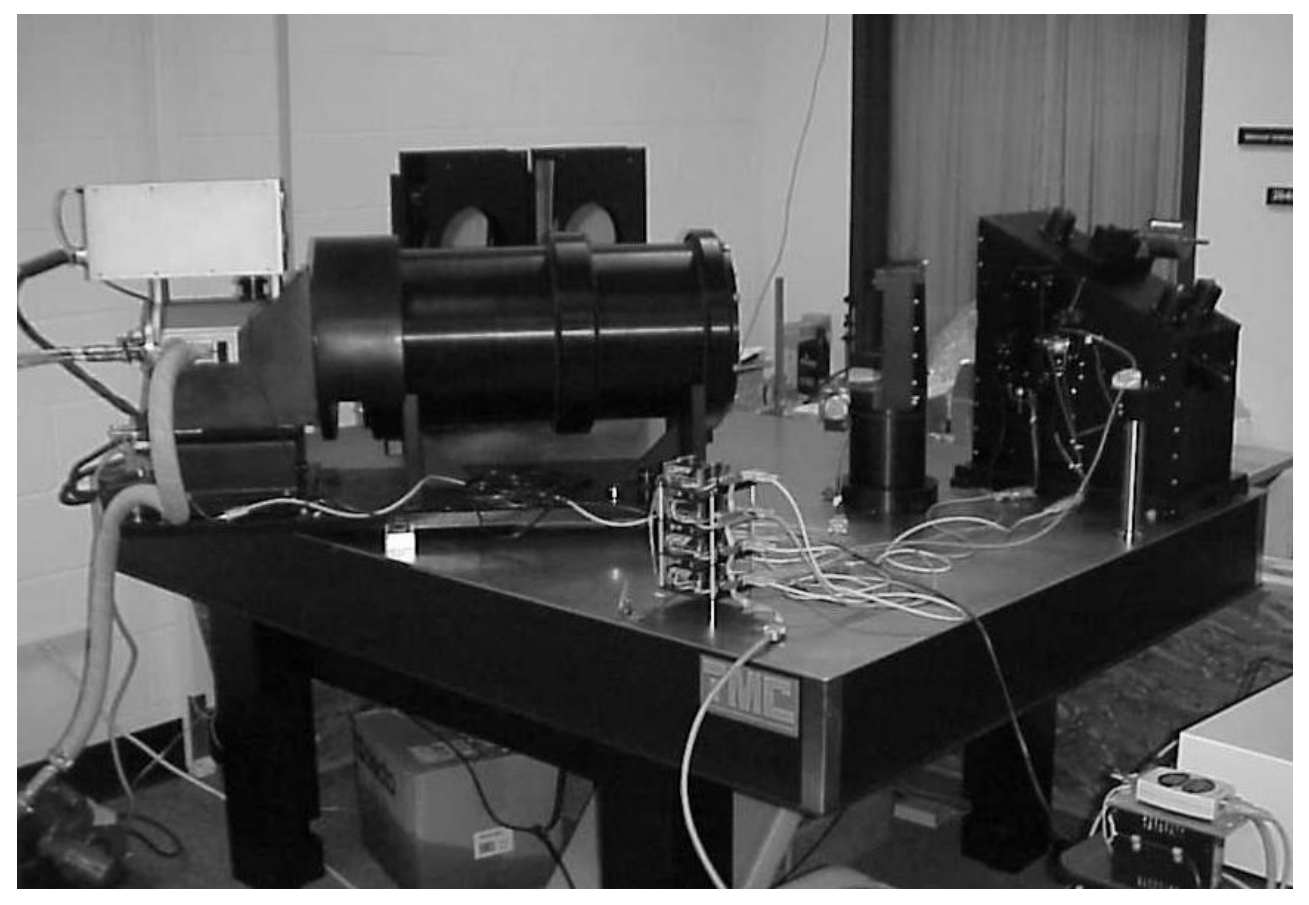

Figure 4. The echelle spectrograph for the TSU 2-m automatic telescope assembled in the lab at TSU.

have found the UDP (universal data packet) protocol quite reliable. It is simpler than TCP/IP and does not require our processes to maintain open sockets. The control daemons know how to respond when such a packet is lost and the controlled process does not report in a reasonable time. Having control of different hardware on different computers eliminates conflicting demands on the computers.

\subsection{Telescope motion control}

Because the two-meter is an automatic telescope, the control system must be absolutely reliable and protected against stalling, at least as much as humanly possible. On the other hand, there are many things one would find in a conventional telescope control system that we simply do not need, namely graphical interfaces and real-time indications of performance. To increase reliability, we designed a telescope drive that required a minimum of cables subject to flexure. The drive consists of six servo motors, two for altitude and four for azimuth. These are all physically attached to the telescope fork. The drives for these servos are located in an insulated box on the fork, and the motion controller is in another box on the fork. Thus there are no wires in the servo system, including those for the encoders, subject to flexure. The only devices not physically attached to the fork are 1) motors to run the secondary mirror and guiding head, all in the telescope tube, 2) an absolute encoder for the azimuth and some digital I/O devices to monitor the position of the enclosure and oil pressure for the hydrostatic azimuth bearing, located in the telescope base and shed for the oil pump. The computer that runs the telescope motions and acquires and tracks stars, t13a, is physically located in the box on the telescope fork with the motion controller. All the cables to the moving part of the telescope, including four power cables, are thus contained in an umbilical about two inches in diameter.

Because the two-meter is an alt-azimuth telescope, it must move continuously in both azimuth and tilt at varying rates. We use an industrial controller to effect these motions, specifically a Galil DMC-2020 twoaxis device with serial communications (RS232 at 38,400 BAUD) to the telescope-control computer. It runs a conventional PID control algorithm with certain filters with parameters set to give stable tracking and slewing. Telescope control obviously introduces an added level of complexity beyond industrial machine control in that the telescope must point to a particular direction at a particular time. Thus we must know both the time and 


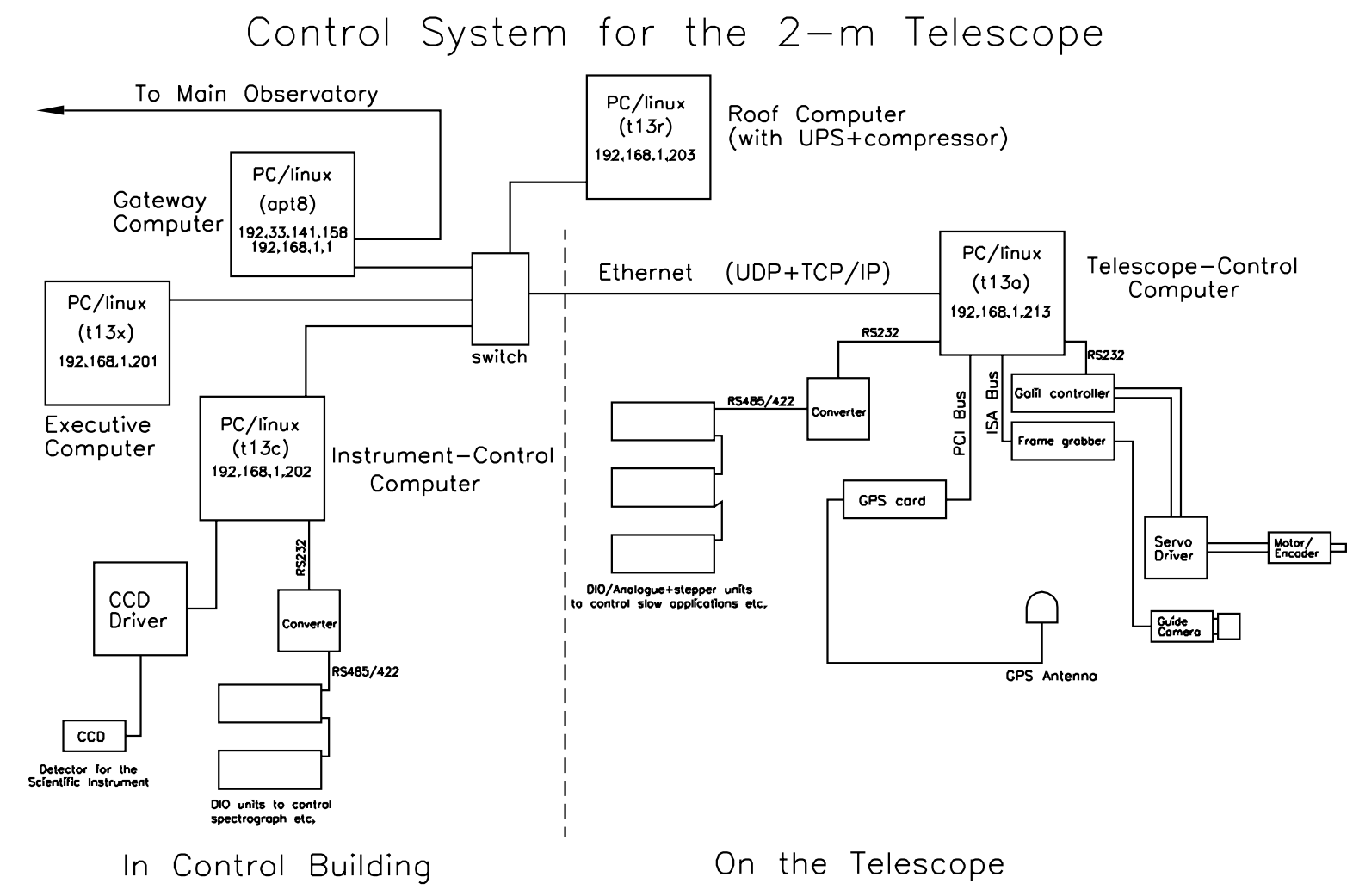

Figure 5. Computers used for controlling the spectroscopic telescope.

the positions of telescope axes very accurately. For a timebase, we use a GPS receiver, specifically a PCI card in t13a, with an antenna on top of the telescope fork. For positions, we count the output of incremental encoders on the two axes in the Galil controller. Knowing the position of the telescope at some time, we calculate speeds for the axes to get the telescope to the position required after a timestep $\Delta t$. The telescope control process does this calculation once every $0.1 \mathrm{sec}$, although we could likely get by with updating it only every second. Of course, the Galil controller updates its PID algorithm much more frequently. We impose two levels of feedback on these rate calculations, one from the mechanically measured positions of the axes and a second from the guide camera. Since we really only need know where the telescope is pointing at some accurately known time, not at a specific time, we use a digital command from the motion controller to latch the positions in the motion controller and time in the GPS receiver (Fig. 6). We then calculate expected positions for the time of these latched positions, correct them for various offsets, then use the differences to adjust the tracking rates sent to the motion controller. Images from the guide camera, taken at rates of up to two a second for bright stars and averaged over roughly ten-second intervals, give a second level of feedback to correct the tracking rates. Deviations calculated in this way show roughly 0.3 -arcsec RMS tracking, quite adequate for our application.

\subsection{Controlling the spectrograph}

We control the spectrograph with another background program running on t13c. It incorporates a linux driver (Astronomical Research Cameras astropci_linux v1.7), written by San Diego State, which we have modified somewhat to run our application. It controls positioning of parts of the spectrograph (choice of input fiber, rotation of secondary grating) and a calibration bench (flip mirror for sources, position of filters to modify color of continuous source, on/off switches for lights) through a serial port to stepper-motor controllers. It communicates with the electronics package for the CCD through a special PCI card driving a two-way serial 


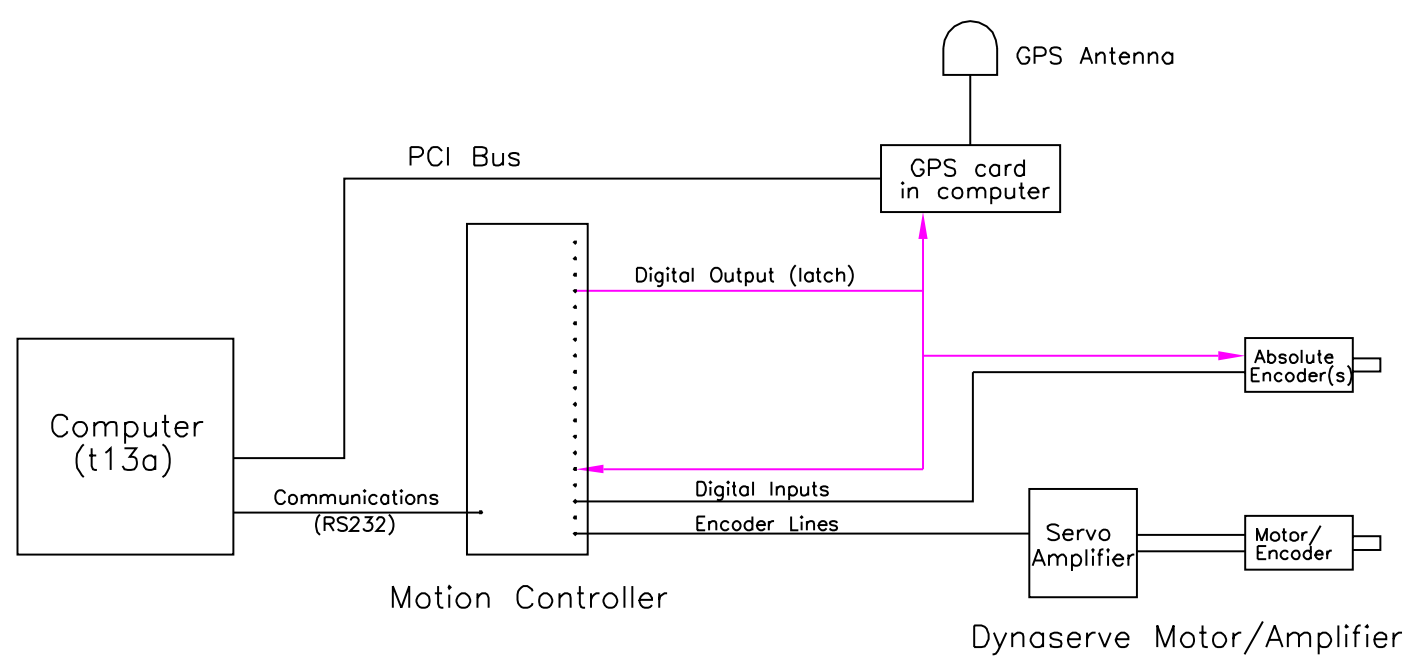

Figure 6. Control scheme for tracking stars. We use the computer t13a to latch positions of the various encoders and time every second.

fiber-optic connection. This card accepts commands through a number of registers above physical RAM. We write to these for such functions as setting the exposure time and binning, choosing an amplifier for the readout, starting the exposure, and reading the temperature for the CCD. The card also fetches the data for an image back through the serial connection, then stores them in a reserved region of computer RAM for analysis or writing to a data file by the control program.

\subsection{Controlling the buildings}

The only building controls that concern the control system are the motors to open and shut the telescope enclosure. This enclosure is a barn-like structure with a base about 7 feet tall, which keeps out most animals, either four-legged or two, and a top that runs off to the north on railroad rails. We use roller chain attached to a DC motor through a worm drive and sprockets to move this structure, a scheme we adapted from Mark Trueblood. The front wall of the building is hinged and flops down for observing under control of two pneumatic cylinders. The mechanical drive system for the building was designed by Louis J. Boyd, and its control program was initially written by Don Epand. Roof control is through a daemon running on computer t13r. It receives commands to open or shut from t13x (by writing files to t13r's disc) and executes them. The control program senses the position of the roof with a multi-turn potentiometer and adjusts the drive rate to keep the roof from banging shut. An optical sensor wired directly into the DC motor controller keeps the roof from moving unless the telescope is in its stowed position. Naturally, the roof motion is protected with limit switches, and motion of the telescope itself is protected by redundant switches that sense whether the enclosure is open. This roof computer also monitors the uninterruptable power supply for the 2-m observatory, located in an auxiliary building north of the telescope enclosure. In two years of operations, the building drive has run reliably except for a few very humid nights when the optical sensor iced up and would not detect the telescope until the sun warmed it.

\subsection{Logging}

To keep track of the proper functioning of the instruments, we write extensive logfiles that can be studied to determine whether something is failing. The executive computer t13x keeps track of when the control system opens and shuts the enclosure, which stars it orders the telescope to observe, whether the telescope acquires them, and when the tracking fails. The telescope computer t13a logs the position of the telecope, pointing errors, and the demand on the servos every 3 seconds. It also logs once a minute the status of the oil pump and enclosure as well as the temperature and humidity in the observatory. Finally, it logs errors about such 
things as finding and tracking stars and slipping drives. The spectrograph computer t13c logs temperatures of the CCD, telescope control room, and spectrograph enclosure every minute. It also records times at which it starts observations, when it reads out the CCD, and when it records exposures in image files. Other information logged by the control programs includes errors of pointing while acquiring stars, which we could use for improving the telescope mount model, and images of starfields when the telescope did not find a star, which are useful for assessing the weather and problems with telescope focus. We also record an infrared image from a weather satellite at midnight, which gives a reasonable picture of the transparency for the night.

We also E-mail to Nashville the nightly log from the roof computer telling when it opened and shut the telescope enclosure, which is good for assessing the weather for the night.

\section{MANAGING THE OBSERVATORY}

There are two aspects of managing the observatory worth mentioning: First, operating the telescope manually for tests. Second, monitoring the performance of the telescope on a daily/weekly basis.

\subsection{Manual operation}

For running the telescope manually, we have written the control programs so that they can run in the foreground on their various computers and accept directions from the command line of a (X-windows) terminal, although the executive daemon requires a separate program tel_control_front to receive commands for it. All the data normally saved into a logfile then show up on the terminal for perusal. This mode is useful for testing changes in the telescope-control software. We also have a video line from the acquisition/guide camera to a monitor in the control room to look at the field of view of the telescope. This setup has proved effective for developing software and analyzing problems when we are working in the control room at the observatory, but it is also possible to use it over the Internet from Nashville.

\subsection{Monitoring telescope performance}

To keep track of the data and detect problems with the telescope, we have developed tools to display pertinent information logged by the control system. We use a quality-control program, qc_t13, that parses the files from the executive, telescope, and spectrograph computers and presents the data collected as either graphs, numerical assessments, or printed error messages. We have three classes of this information, 1) environmental data, such as humidity outdoors and temperature at key places in the observatory, 2) a record of stars acquired, not found, or lost by the telescope, 3) extensive information about tracking, such as demand on servos, tracking errors, and offsets of stars from the nominal center of the field of the telescope, which we present graphically and in some instances as averages for the night. This program lets us look at the images of acquisition fields when the telescope did not find a star for assessing problems with weather, pointing, or focusing. It also prepares a summary of stars observed for the night which is useful in updating the target list for subsequent nights. A similar quality-control program gives us an assessment of the quality of the spectra for a night.

\section{TOOLS FOR SCHEDULING AND DATA HANDLING}

Naturally we have developed extensive tools for copying data back to TSU, for reducing the data to spectra, for simulating the telescope's operation, and for updating observing programs to keep the frequencies of observation roughly proportional to the desired rates.

\subsection{Copying logfiles and data}

We use cron jobs to reduce the spectra automatically at the end of the night and to collect the various logfiles on the gateway computer, apt8, for copying back to Nashville. The files for a night always have roughly the same names, so it is easy to copy blocks of them back to Nashville with secure copy, scp, for example, by using wildcards to specify file names. Data for a given night are recorded in subdirectories identified with the Modified Julian Date for that night, so the files for a given night can easily be distinguished from other files with the same names for other nights. We have separate programs to copy the various logfiles from the gateway computer and files from the reduction of the spectra from the spectrograph computer. 


\subsection{Updating the observing program}

The program that parses the logfiles, qc_t13, creates a summary file for the night which can be used to update the observing priorities for a given program in light of the observations recently made. We do this with a program freq_t13 that reads the standard starlist for an observing program (or night), looks back a specified number of days for observations of stars in that program, adjusts the priorities for observations to favor stars that have not been observed as frequently as desired, and writes a new observing list with these adjusted priorities. It thus makes it possible to observe all the stars in a program at roughly the desired frequencies. We can then copy the new observing list back to the executive computer at the observatory, as we had copied the data to Nashville.

\subsection{Archiving the reduced spectra}

The reduced spectra are contained in FITS files consisting of an array of wavelengths and intensities for a certain number of 2048-pixel orders of the echelle spectra. These have to be archived and knitted together to get rid of the order structure. To do this, we 1) copy the data back from the observatory as explained above, run a quality-control program to look at the quality of the spectra, 3) renormalize the various orders and map them onto a common wavelength scale, and 4) make a record of each final reduced spectrum in a database.

\section{EXPERIENCE TO DATE}

The AST began tracking stars in October, 2001, obtained its first spectroscopic data in May, 2003, and has been observing regularly since late September, 2003.

\section{ACKNOWLEDGMENTS}

There are a number of people who have contributed materially to this project and have been particularly helpful or professional. First among them is Harland Epps whose experience and technical skills were indispensible for completing our spectrograph. Our Center Director, Michael R. Busby, managed all the contracts and solved the extensive purchasing problems this project presented a relatively small state university. Alan Schier designed the physical mount for the spectrograph camera, had the pieces manufactured, then assembled it so the camera worked out of the box. Jeff Hall of Lowell Observatory wrote the computer program to reduce the data, and Louis J. Boyd of Fairborn Observatory designed and built the controls for the telescope enclosure. Several TSU students worked on early parts of the control system and later on the software for scheduling, quality-control, and simulation.

\section{REFERENCES}

1. J. A. Eaton, G. W. Henry, and F. C. Fekel, "Advantages of Automated Observing with Small Telescopes," The Future of Small Telescopes In The New Millennium. Volume II - The Telescopes We Use, T.D. Oswalt, ed., pp. 189-207, Kluwer, Dordrecht, 2003.

2. K. G. Libbrecht and M. L. Peri, "A Fiber-Fed Echelle Spectrograph for the Hale 5-m Telescope," Publ. Astr. Soc. Pacific, 107, pp. 62-67, 1995.

3. H. Dekker, B. Delabre, G. Hess, and H. Kotzlowski "The UV-Visual Echelle Spectrograph for the VLT (UVES)," in Progress in Telescope and Instrumentation Technologies, M.-H. Ulrich, ed., p. 581, ESO, Garching, 1992.

4. C. Pilachowski, H. Dekker, K. Hinkle, R. Tull, S. Vogt, D. D. Walker, F. Diego, and R. Angel, "HighResolution Spectrographs for Large Telescopes," Publ. Astr. Soc. Pacific, 107, pp. 983-989, 1995.

5. H. W. Epps, "Development of large high-performance lenses for astronomical spectrographs," in Optical Astronomical Instrumentation, S. D'Odorico, ed., Proc. SPIE 3355, pp. 111-128, 1998.

6. L. J. Boyd, private communication, 2002. 